\title{
The effects of Commiphora mukul extract on spermatogenesis and testosterone levels in male diabetic rats
}

\author{
Ali Akbar Rezaei ${ }^{1,2}$, Iraj Salehi', Seyed Asaad Karimi ${ }^{1,3}$, Mehdi Rahnama ${ }^{2}$ \\ ${ }^{1}$ Neurophysiology Research Center, Hamadan University of Medical Sciences, Hamadan; ${ }^{2}$ Department of Physiology, Faculty of Science, Science and \\ Research Branch, Islamic Azad University, Zanjan; ${ }^{3}$ Department of Neuroscience, School of Science and Advanced Technologies in Medicine, Hamadan \\ University of Medical Sciences, Hamadan, Iran
}

Objective: The strong antioxidant activity of Commiphora mukul prompted us to conduct the present study to explore whether treatment with C. mukul extract (CME) would have any protective influence on sperm parameters, testosterone levels, and plasma glucose levels in streptozotocin (STZ)-induced diabetic rats.

Methods: Male Wistar rats were randomly divided into four groups: control, control animals treated with CME, diabetic animals, and diabetic animals treated with CME. CME extract ( $300 \mathrm{mg} / \mathrm{kg}$ ) was administered for 60 days by daily gavage. Diabetes was induced by an intraperitoneal injection of $50 \mathrm{mg} / \mathrm{kg} \mathrm{STZ}$. The epididymal sperm count, weight, motility, morphology, viability, and serum testosterone and glucose levels were determined.

Results: In the diabetic animals, CME decreased blood glucose levels $(p<0.05)$, increased the total sperm count $(p<0.05)$, and decreased the proportion of sperm with abnormal morphology $(p<0.05)$. Diabetes reduced sperm motility $(p<0.001)$, and CME supplementation partially reversed this effect of diabetes $(p=0.003)$. Furthermore, in diabetic animals, CME decreased the proportion of immotile sperm $(p<0.001)$. In rats, diabetes caused a significant decrease $(p<0.05)$ in serum testosterone levels $(F[3,28]=3.283, p=0.035)$, but treatment of diabetic animals with CME increased serum testosterone levels.

Conclusion: The present study demonstrated that $C$. mukul possesses proandrogenic activity and exerts a beneficial effect on sperm parameters in diabetic rats.

Keywords: Commiphora mukul; Diabetes mellitus; Sperm; Streptozotocin; Rats

\section{Introduction}

Diabetes mellitus (DM) is a chronic metabolic disease characterized

Received: August 4, 2019· Revised: September 16, 2019· Accepted: September 29,2019

Corresponding author: Seyed Asaad Karimi

Department of Neuroscience, School of Science and Advanced Technologies in Medicine, Hamadan University of Medical Sciences, Hamadan 6516665178, Iran Tel:+98-910-778-5147 Fax:+98-81-38380208 E-mail:asad_karimi_128@yahoo.com

*This research was supported by a grant from the Hamadan University of Medical Sciences, Hamadan, Iran.

This is an Open Access article distributed under the terms of the Creative Commons Attribution Non-Commercial License (https://creativecommons.org/licenses/by-nc/4.0/) which permits unrestricted non-commercial use, distribution, and reproduction in any medium, provided the original work is properly cited. by hyperglycemia and dysfunctional glucose and lipid metabolism that results from insufficient or defective insulin secretion, insulin resistance, or both [1]. DM can exert destructive effects on multiple organs in the body, and especially on male infertility [2]. DM and metabolic syndrome affect sperm quality and function and may cause retrograde ejaculation and hypogonadism [3]. Furthermore, DM can interrupt the endocrine control of spermatogenesis [4].

Insulin resistance and/or impaired insulin secretion in DM results in the overproduction of reactive oxygen species (ROS), leading to oxidative stress that triggers diabetic complications [5]. It has been reported that spermatozoa are susceptible to oxidative stress-induced damage [6]. Previous studies have shown that men with DM have a higher proportion of spermatozoa with nuclear DNA damage, result- 
ing in alterations of male fertility, patterns of early embryonic growth, reproductive outcomes, and miscarriage rates [7-10]. Since oxidative stress damages spermatozoa, antioxidants and medicinal herbs play an important role in the maintenance of sperm motility and sperm DNA integrity in the face of oxidative damage [11-13]. Plants have always been an exemplary source of drugs, and many currently available drugs have been derived from plants.

Commiphora mukul (in the Burseraceae family), commonly known as gum guggulu, has been used for over 2,500 years as a valued herb in Ayurvedic Medicine, a system of South Asian Medicine practiced in India, Bangladesh, and Pakistan. Bellamkonda et al. [14] reported the antihyperglycemic and antioxidant activities of an alcoholic extract of $C$. mukul gum resin in streptozotocin (STZ)-induced diabetic rats. $C$. mukul has the potential to promote health in individuals with DM and many other diseases through its anti-inflammatory, hypoglycemic, antihyperlipidemic, and antioxidant effects $[14,15]$.

Given the antioxidant effects of C. mukul and the fact that DM is associated with enhanced oxidative stress and diminished antioxidant status, our hypothesis was that C. mukul would act as a protective factor against the negative consequences of DM. The strong antioxidant activity of $C$. mukul prompted us to design the present study to explore whether treatment with C. mukul extract (CME) would have any protective influence on sperm parameters, testosterone levels, and plasma glucose levels in STZ-induced diabetic rats.

The following MeSH terms for C. mukul were used in our search of the literature: Commiphora molmol, Commiphora wightii, Commiphora myrrha, Commiphora molmol, Common Myrrh Tree, Myrrh Tree, Common Tree, Common Myrrh, Guggul Tree, Commiphora erythraea, Myrrh, Bisabol, Commiphora kataf, Bisabol Myrrh, and Guggul.

\section{Methods}

\section{Ethics statement}

All experimental procedures using rats were conducted in accordance with the Animal Care and Use Guidelines approved by the Institutional Ethics Committee of Hamadan University of Medical Sciences and were performed in accordance with the National Institutes of Health Guide for Care and Use of Laboratory Animals [16]. All efforts were made to minimize suffering. Operations that could cause pain and distress were performed in a separate room in the absence of other animals.

\section{Animals and experimental design}

Adult male Wistar rats weighing $220 \pm 10 \mathrm{~g}$ were obtained from the Pasteur Institute of Tehran, Iran. The animals were housed in an airconditioned room at $22^{\circ} \mathrm{C} \pm 2{ }^{\circ} \mathrm{C}$ with a 12-hour light/dark cycle. The animals were kept in cages with $2-3$ rats in each cage. Standard animal chow and water were freely available. After 1 week of adaptation, the subjects were randomly divided into four groups: control, control animals treated with CME (300 mg/kg), diabetic animals, and diabetic animals treated with CME $(300 \mathrm{mg} / \mathrm{kg})$. The oral dosage of CME was selected based on our previous work [17], in light of the finding that a dose of less than $200 \mathrm{mg} / \mathrm{kg}$ body weight is not expected to be effective in rats [14]. All rats had free access to food and tap water. Saline or CME was administered for 60 days by daily gavage through gastric intubation using a syringe once daily at 8:00 AM. The experimental design of this study is presented in Figure 1.

\section{Induction of diabetes}

Diabetes was induced by an intraperitoneal injection of $50 \mathrm{mg} / \mathrm{kg}$

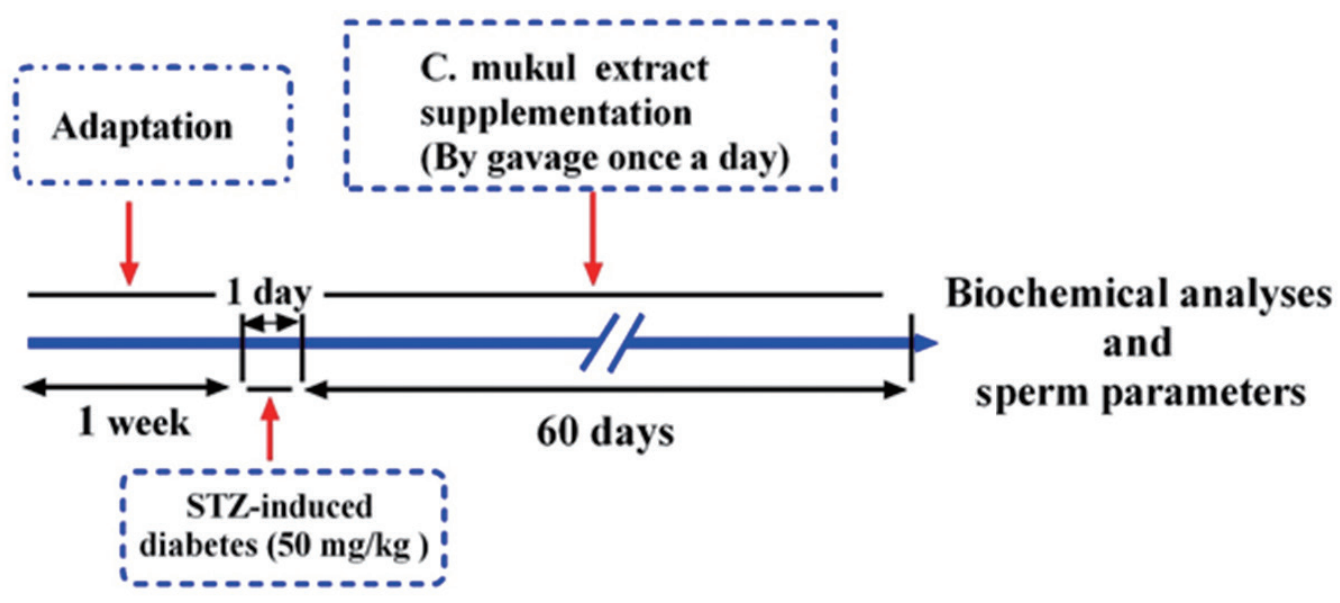

Figure 1. Experimental design. After 1 week of adaptation, Commiphora mukul (C. mukul) extract (300 mg/kg) was administered intragastrically by gavage once a day for 60 days. Epididymal sperm parameters and biochemical parameters were then analyzed in male rats. STZ, streptozotocin. 
STZ (Sigma, St. Louis, MO, USA), which was dissolved in freshly prepared $0.05 \mathrm{M}$ citrate buffer ( $\mathrm{pH} 4.5$ ) immediately before injection $[18,19]$. Blood glucose concentrations were monitored once per week. A minimum blood glucose level of $>250 \mathrm{mg} / \mathrm{dL}$ and the presence of urinary glucose were used as criteria to identify diabetic rats. Age-matched, vehicle-treated rats were used as controls. Serum fasting blood glucose concentrations were measured using the glucose oxidase method (Pars Azmoon kits; Tehran, Iran); the intra- and interassay coefficients of variation were $2.5 \%$ and $6.1 \%$, respectively [20].

\section{Preparation of ethanolic extract of $C$. mukul}

An ethanolic extract of $C$. mukul gum resin was obtained from the manufacturers and exporters of herbal extracts. The following procedure was used to prepare the extract: the collected plant sample (resin) was washed thoroughly with tap water, dried at room temperature away from sunlight, cut into small pieces, and then powdered. Ethanolic extract was prepared by cold maceration of gum resin powder in ethanol for 7 days. The extract was filtered, concentrated under reduced pressure, and finally dried in a vacuum desiccator. The extract was stored at $0^{\circ} \mathrm{C}-4^{\circ} \mathrm{C}$ and dissolved in water just before use [21].

\section{Epididymal sperm count, weight, motility, morphology, and viability}

\section{1) Sperm collection and sperm count}

A laparotomy was performed to expose the reproductive tract. Testis weight and the ratio of testis weight to body weight were determined. The left and right epididymis were carefully separated from the testis and placed in a Petri dish containing Ham's F10 medium. The epididymides were minced with scissors to release sperm and were then placed in an incubator for 15 minutes. Approximately 10 $\mu \mathrm{L}$ of the diluted sperm suspension was transferred to each counting chamber of the hemocytometer and allowed to stand for 5 minutes. The cells that settled during this time were counted under light microscopy at $\times 200$ magnification. The sperm heads were counted and expressed as millions $/ \mathrm{mL}$ of suspension [22].

\section{2) Sperm morphology}

Sperm morphology was also characterized using eosin and nigrosin staining as described elsewhere [23,24]. To a test tube containing $40 \mu \mathrm{L}$ of sperm suspension, $10 \mu \mathrm{L}$ of $1 \%$ eosin $\mathrm{Y}$ and nigrosin was added and mixed by gentle agitation. Sperm were incubated at room temperature for 45-60 minutes for staining and then re-suspended with a Pasteur pipette. It is preferable to examine 200 sperm per animal at $\times 400-1,000$ magnification for morphological analyses. Morphological abnormalities were classified as follows: headless sperm, flattened head (reduced hook, banana head, pin, or nail), bent neck, bent tail, kinked tail, or multiple abnormalities [24].

\section{3) Sperm motility}

Spermatozoa were classified as motile or immotile. Aliquots of the sperm suspension prepared for analysis were placed on a slide. The slides were evaluated with phase contrast microscopy in 10 microscopic fields, and 200 sperm per animal were analyzed at a final magnification of $\times 1,000$. The motile sperm fraction was defined as the mean number of motile sperm $\times 100$, divided by the total number of sperm [23].

\section{4) Sperm viability}

Sperm viability was also determined by using eosin staining (Sigma Aldrich, St. Louis, MO, USA) as described previously [24]. Eosin penetrates non-viable, dead spermatozoa with disrupted membranes, which stain red using this technique. The percentage of normal morphology per 100 spermatozoa for each rat was assessed under light microscopy ( $\times 400$; Zeiss, Munich, Germany) as described previously $[22,24]$. An experienced technician blinded to the study performed all analyses.

\section{Serum testosterone measurements}

After blood collection from the heart, specimens were centrifuged. Then, the serum concentration of testosterone was measured using an enzyme-linked immunosorbent assay kit for testosterone (Demeditec, Kiel, Germany) [13]. The sensitivity of hormone detection in each assay tube was $0.066 \mathrm{ng} / \mathrm{mL}$.

\section{Statistical analysis}

All data are presented as mean \pm standard error of the mean. Statistical analyses were performed using GraphPad Prism (v. 8.0; GraphPad Software, La Jolla, CA, USA). The distribution of data was normal when examined using the Kolmogorov-Smirnov test. One-way analysis of variance (ANOVA) was performed, followed by the Tukey test for multiple comparisons. A $p$-value of 0.05 was considered as the criterion for significance.

\section{Results}

\section{Effects of DM and CME on blood glucose}

Mean blood glucose levels were analyzed before and after the STZ injection and at the end of experiment (Figure 2). Before the STZ injection, there was no significant difference among the experimental groups of rats $(F[3,16]=0.833, p=0.495$, one-way ANOVA). After the STZ injection, the mean blood glucose levels in the DM and DM+ CME groups were significantly higher than in the control group ( $F[3$, $16]=130.1, p<0.001$, one-way ANOVA). 


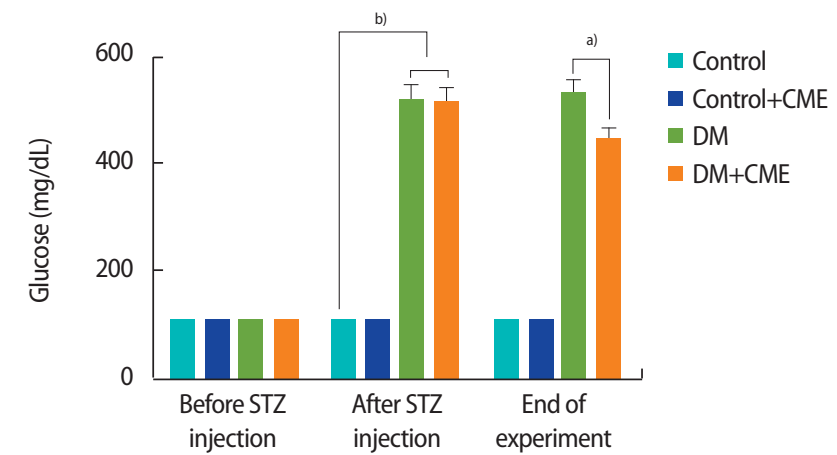

Figure 2. Mean blood glucose levels. Glucose levels were measured before and after the streptozotocin injection and at the end of the experiment. Each column and bar represents mean \pm standard error of the mean. CME, Commiphora mukul extract; DM, diabetes mellitus; STZ, streptozotocin. ${ }^{\text {al }} p<0.05$; ${ }^{\text {b }} p<0.001$.

At the end of the experimental period, venous blood was collected from all rats and mean blood glucose levels were analyzed ( $F[3$, $16]=178.7, p<0.001$, one-way ANOVA]. Our results showed that CME supplementation decreased blood glucose levels in animals with DM $(p<0.05)$. The results are summarized in Figure 2.

\section{Effects of DM and CME on testis weight and the ratio of testis weight to body weight}

Figure 3 shows the effect of DM and CME treatment on testis weight and the ratio of testis weight to body weight. Significant differences were found among the experimental groups. The final body weight was significantly different among the experimental groups $(F[3,16]=440.1, p<0.001$, one-way ANOVA). Figure $3 \mathrm{~A}$ shows the final body weight of the rats in each group. The body weight of all diabetic animals was significantly lower than the body weight of the control group $(p<0.001)$. The DM+CME group demonstrated a higher final body weight than the DM group ( $p<0.01)$, and also exhibited more weight gain than the DM group. Figure $3 \mathrm{~B}$ shows the testis weight of the rats in each group. Testis weight was significantly different among the experimental groups $(F[3,16]=400.2, p<0.001$, one-way ANOVA). The DM group exhibited lower testis weight than the control group $(p<0.001)$. In the DM+CME group, testis weight was higher than that of the DM animals without CME $(p<0.01)$. The ratio of testis weight to body weight did not significantly differ among the experimental groups $(p>0.05)$ (Figure 3C). The results are summarized in Figure 3.

\section{Effects of DM and CME on sperm parameters}

Figure 4 shows the effects of DM and CME treatment on epididymal sperm characteristics. The total sperm count extracted from the epididymis decreased significantly in diabetic animals compared with the control group $(F[3,28]=10.01, p=0.001$, one-way ANOVA)
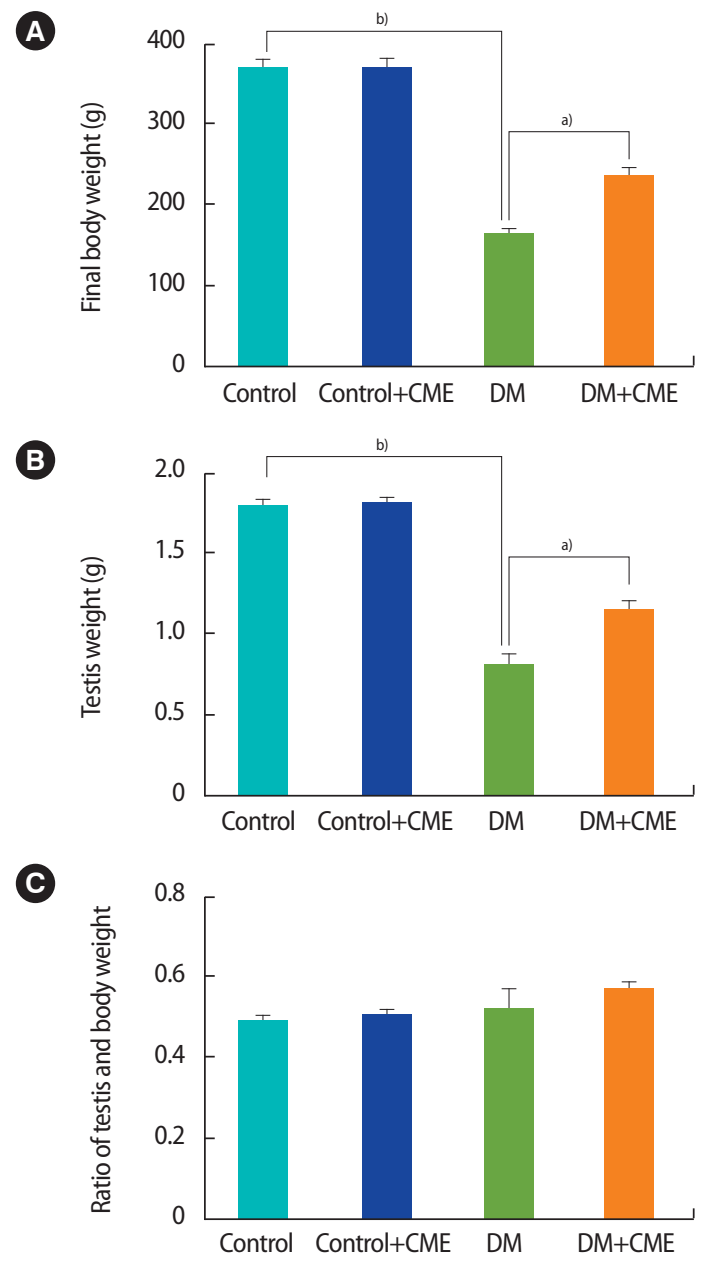

Figure 3. Body weight (A), testis weight (B), and the ratio of testis weight to body weight (C). Each column and bar represents mean \pm standard error of the mean. CME, Commiphora mukul extract; DM, diabetes mellitus. ${ }^{\text {a) }} p<0.01$; ${ }^{\text {b) }} p<0.001$.

(Figure 4A). Treatment of diabetic animals with CME increased the total sperm count in the DM animals $(p<0.05)$. Sperm viability decreased significantly in the DM group in comparison with the control group $(F[3,28]=3.062, p=0.044$, one-way ANOVA) (Figure 4B). There was no significant difference between the control group and the DM + CME animals $(p>0.05)$.

The percentage of sperm with normal morphology was lower in the animals with DM than in the control animals $(F[3,28]=4.756, p=0.008$, one-way ANOVA) (Figure 4C). In addition, the proportion of sperm with abnormal morphology was higher in the animals with DM than in the control animals $(F[3,28]=4.750, p=0.008$, one-way ANOVA) (Figure $4 C$ ). The proportion of sperm with abnormal morphology was lower in the DM+CME group than in the DM group $(p<0.05)$.

Figure $4 \mathrm{D}$ shows that sperm motility was significantly different among the groups $(F[3,28]=15.32, p<0.001$, one-way ANOVA]. DM reduced sperm motility $(p<0.001)$ and CME supplementation par- 
A

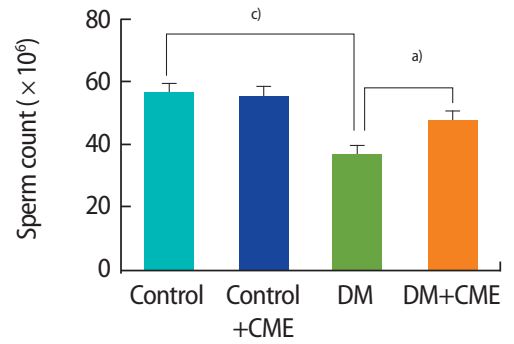

C

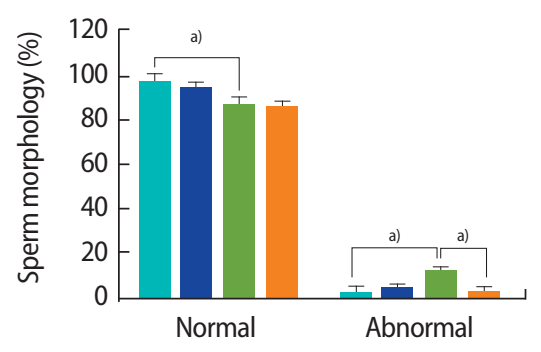

E

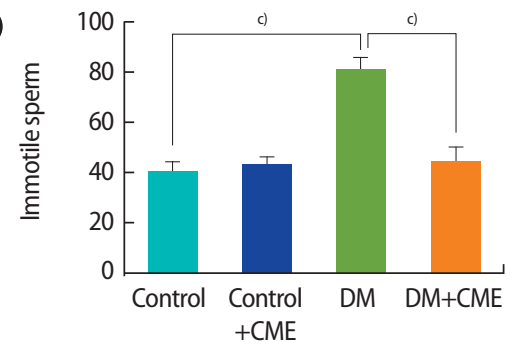

B

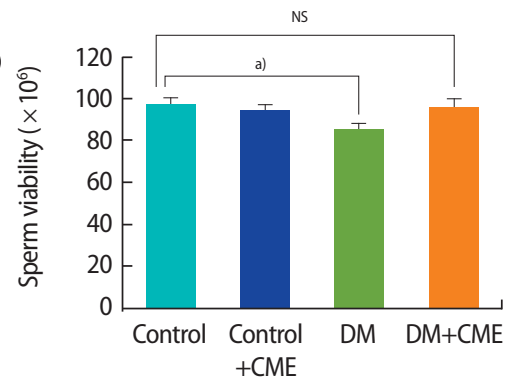

- Control

- Control+CME

- DM

- $\mathrm{DM}+\mathrm{CME}$

D

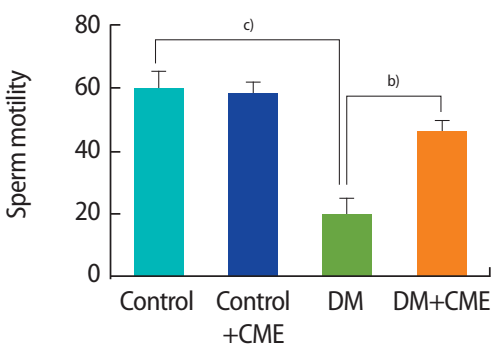

F

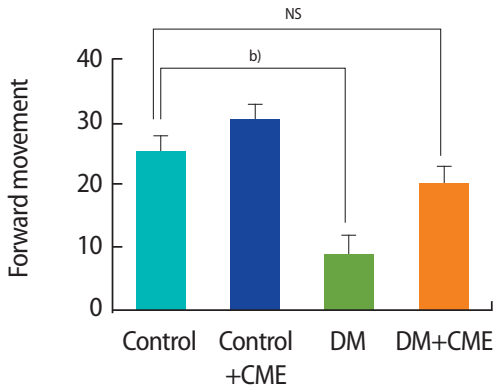

Figure 4. Sperm parameters: (A) sperm count, (B) viability, (C) morphology, (D) motility, (E) immotile sperm, and (F) forward movement in the experimental groups. Each column and bar represents mean \pm standard error of the mean. CME, Commiphora mukul extract; DM, diabetes mellitus; NS, not significant. ${ }^{\text {a) }} p<0.05 ;{ }^{\text {b) }} p<0.01 ;{ }^{\text {c) }} p<0.001$.

tially reversed this effect $(p=0.003)$. Moreover, the percentage of immotile sperm was higher in the animals with DM $(F[3,28]=14.75$, $p<0.001$, one-way ANOVA) (Figure 4E). Treatment of diabetic animals with CME decreased the proportion of immotile sperm $(p<$ 0.001). Furthermore, forward movement was reduced in the DM group $(F[3,28]=8.963, p<0.001$, one-way ANOVA) (Figure 4F). All results are summarized in Figure 4.

\section{Effects of DM and CME on blood testosterone levels}

The mean serum testosterone levels in the DM and control animals were $0.7 \pm 0.2$ and $1.75 \pm 0.2 \mathrm{ng} / \mathrm{mL}$, respectively. Thus, it was clear that DM in rats caused a significant decrease $(p<0.05)$ in serum testosterone levels compared to the control group $(F[3,28]=3.283$, $p=0.035$, one-way ANOVA) (Figure 5). However, there was no significant difference between the DM+CME group and the control animals $(p=0.995)$.

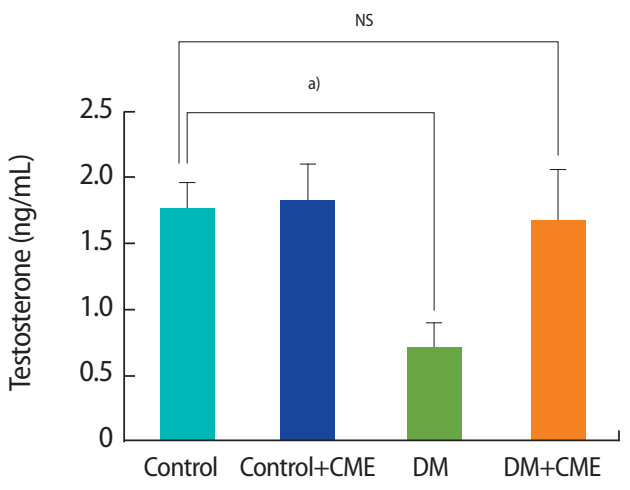

Figure 5. Mean serum testosterone levels in the experimental groups. Each column and bar represents mean \pm standard error of the mean. NS, not significant; CME, Commiphora mukul extract; DM, diabetes mellitus. ${ }^{\text {a) }} p<0.05$. 


\section{Discussion}

This study was undertaken to investigate whether CME could attenuate the negative effects of DM on sperm parameters, testosterone levels, and plasma glucose levels in STZ-induced diabetic rats. Sperm motility, count, and viability were significantly lower in diabetic animals than in the control group. We also showed that the rates of morphological abnormalities in sperm were considerably higher in diabetic animals. However, CME-treated diabetic rats had significantly higher proportions of normal sperm morphology, motility, count, and viability than non-CME-treated diabetic rats. Moreover, C. mukul attenuated the adverse effects of DM on testosterone levels. In addition, C. mukul supplementation decreased blood glucose levels in diabetic animals.

In accordance with our results, it has been shown that DM and hyperglycemia pose a threat to spermatozoa function and can increase the risk of infertility $[25,26]$. The impact of DM on the male reproductive system may be due to its effects on endocrine control of spermatogenesis, steroidogenesis, sperm maturation, and penile erectile dysfunction [27].

Insulin resistance and/or impaired insulin secretion in DM result in hyperglycemia. Persistent hyperglycemia induces the overproduction of ROS, the formation of advanced glycation end products, secretion of proinflammatory cytokines and cellular death, and, consequently, diabetic complications [5]. Karimi et al. [28] showed that increased ROS production altered sperm parameters and increased sperm nuclear DNA fragmentation. Shrilatha et al. [29] also reported STZ-induced oxidative damage in the testis and epididymal sperm of mice.

Since oxidative damage leads to many problems, it has become increasingly common to use antioxidant agents and medicinal herbs to prevent and treat various types of diabetes-related disorders $[12,30,31]$. For example, Bahmanzadeh et al. [12] showed that dietary supplementation with astaxanthin may ameliorate sperm parameters and DNA integrity in STZ-induced diabetic rats.

Some bioactive compounds has been reported in $\mathrm{CME}$, including dimyrcene; a-camphorene; linoleic, oleic, stearic, and palmitic acids; sitosterol; Z- and E-guggulsterone; and (8R)-3a,8-dihydroxypolypoda-13E,17E,21-triene (myrrhanol C,4-pregnene-3,16-dione, 20S-acetyloxy-4-pregnene-3,16-dione, 4,17(20)-(cis)-pregnadiene-3,16-dione, 4,17(20)-(trans)-pregnadiene-3,16-dione, 16 $\beta$-acetyloxy-pregn-4,17(20)-trans-dien-3-one, 3 a-acetyloxy-5apregnan-16-one, 20R,22R-dihydroxycholest-4-en-3-one) [32,33]. Furthermore, several amino acids have been reported to be present in $\mathrm{CME}$, including cystine, histidine, alanine, proline, tyrosine, tryptophan, valine, leucine, and isoleucine [33]. Commipheric acid, guggulipid (ethyl acetate extract of the gum of the guggul tree), and gug- gulsterone are the main components of C. mukul (guggul) [34]. Guggul is reported to have antidiabetic properties and it acts as a dual activator for peroxisome proliferator-activated receptors alpha and gamma (PPAR- $\alpha$ and PPAR- $\gamma$ ) $[34,35]$. Guggulsterone has been reported to exert both hypoglycemic and hypolipidemic activity in high-fat diet-induced diabetic rats, and it may have therapeutic potential for type 2 DM [36]. Bellamkonda et al. [14] showed beneficial effects of ethanolic CME against high-fructose diet-induced abnormalities in carbohydrate and lipid metabolism in Wistar rats. They found that 60 days of $C$. mukul treatment completely prevented fructose-induced increased body weight, hyperglycemia, insulin resistance, and hypertriglyceridemia [37]. It has also been reported that ethanolic CME attenuated STZ-induced alterations in the activity of enzymes involved in glucose metabolism (hexokinase, phosphofructokinase, pyruvate kinase, glucose-6-phosphatase, fructose-1,6bisphosphatase, and glucose-6-phosphate dehydrogenase) and lipid metabolism (fatty acid synthase, malic enzyme, and lipoprotein lipase) [21]. Ramesh et al. [38] showed that C. mukul played a preventive role against STZ-induced diabetic oxidative stress. They reported that the administration of C. mukul ( $200 \mathrm{mg} / \mathrm{kg}$ body weight) to diabetic rats for 60 days decreased the activity of aspartate aminotransaminase and alanine aminotransaminase in the liver and kidney and reduced the levels of oxidative markers such as lipid peroxidation and protein oxidation in the pancreas and heart.

CMEs have been extensively studied for their broad range of biological activities, especially related to their antioxidant properties. The antioxidant activity of an alcoholic extract of $C$. mukul gum resin in STZ-induced diabetic rats was reported by Ramesh et al. [38]. In addition, it has been shown that $C$. molmol attenuated oxidative stress in hyperammonemic rats [39]. It was found that C. mukul significantly lowered lipid peroxidation by maintaining the activity of the major antioxidant enzymes (superoxide dismutase, catalase and glutathione peroxide) in STZ-induced diabetic rats [14]. Cellular damage in semen is the result of an inappropriate balance between ROS production and scavenging activities. Extreme ROS generation that exceeds critical levels can overwhelm all the antioxidant defense strategies of spermatozoa and seminal plasma, causing oxidative stress $[40,41]$. Because of the high content of polyunsaturated fatty acids in the plasma membrane, spermatozoa are highly sensitive to excessive concentrations of ROS. Lipid peroxidation destroys the structure of the lipid matrix in the sperm membrane, which is accompanied by a loss of motility and impaired spermatogenesis $[42,43]$.

In the present study, the administration of CME for 60 consecutive days significantly increased sperm motility and viability in diabetic animals. This increase in sperm motility and viability may have been due to the protective effects of $C$. mukul. No toxicity symptoms occur 
in response to chronic treatment with C. molmol [44]. Furthermore, it has been reported that C. molmol has androgenic potential [45], and based on our results, it attenuates the negative effects of diabetes on sperm parameters, testosterone levels, and plasma glucose levels in STZ-induced diabetic rats. Several plants have been found to affect serum testosterone and dihydrotestosterone levels [44]. Testosterone and related androgenic derivatives are essential for male fertility and the maintenance of spermatogenesis. In conclusion, the present study demonstrated that $C$. mukul possesses antioxidant and proandrogenic activities, enabling it to exert a beneficial effect on spermatogenesis and sperm parameters in rats.

\section{Conflict of interest}

No potential conflict of interest relevant to this article was reported.

\section{Author contributions}

Conceptualization: IS. Data curation: IS, MR, AAR. Formal analysis: SAK. Funding acquisition: IS. Methodology: IS. Project administration: IS, SAK. Visualization: IS, SAK. Writing - original draft: AAR, SAK. Writing - review \& editing: all authors.

\section{References}

1. American Diabetes Association. 2. Classification and diagnosis of diabetes. Diabetes Care 2016;39 Suppl 1:S13-22.

2. La Vignera S, Condorelli R, Vicari E, D'Agata R, Calogero AE. Diabetes mellitus and sperm parameters. J Androl 2012;33:145-53.

3. Mallidis C, Czerwiec A, Filippi S, O'Neill J, Maggi M, McClure N. Spermatogenic and sperm quality differences in an experimental model of metabolic syndrome and hypogonadal hypogonadism. Reproduction 2011;142:63-71.

4. Sexton WJ, Jarow JP. Effect of diabetes mellitus upon male reproductive function. Urology 1997;49:508-13.

5. Volpe CM, Villar-Delfino PH, Dos Anjos PM, Nogueira-Machado JA. Cellular death, reactive oxygen species (ROS) and diabetic complications. Cell Death Dis 2018;9:119.

6. Agarwal A. Role of oxidative stress in male infertility and antioxidant supplementation. US Kidney Urol Dis 2005;122.

7. Agbaje IM, Rogers DA, McVicar CM, McClure N, Atkinson AB, Mallidis $C$, et al. Insulin dependant diabetes mellitus: implications for male reproductive function. Hum Reprod 2007;22:1871-7.

8. Mangoli E, Talebi AR, Anvari M, Pourentezari M. Effects of experimentally-induced diabetes on sperm parameters and chromatin quality in mice. Iran J Reprod Med 2013;11:53-60.

9. Aitken RJ, Baker MA. Oxidative stress, sperm survival and fertility control. Mol Cell Endocrinol 2006;250:66-9.

10. Lewis SE. Is sperm evaluation useful in predicting human fertility? Reproduction 2007;134:31-40.

11. Hughes CM, Lewis SE, McKelvey-Martin VJ, Thompson W. The effects of antioxidant supplementation during Percoll preparation on human sperm DNA integrity. Hum Reprod 1998;13:1240-7.

12. Bahmanzadeh M, Vahidinia A, Mehdinejadiani S, Shokri S, Alizadeh Z. Dietary supplementation with astaxanthin may ameliorate sperm parameters and DNA integrity in streptozotocin-induced diabetic rats. Clin Exp Reprod Med 2016;43:90-6.

13. Ahangarpour A, Oroojan AA, Radan M. Effect of aqueous and hydro-alcoholic extracts of lettuce (Lactuca sativa) seed on testosterone level and spermatogenesis in NMRI mice. Iran J Reprod Med 2014;12:65-72.

14. Bellamkonda R, Rasineni K, Singareddy SR, Kasetti RB, Pasurla R, Chippada AR, et al. Antihyperglycemic and antioxidant activities of alcoholic extract of Commiphora mukul gum resin in streptozotocin induced diabetic rats. Pathophysiology 2011;18:255-61.

15. Urizar NL, Moore DD. GUGULIPID: a natural cholesterol-lowering agent. Annu Rev Nutr 2003;23:303-13.

16. Institute of Laboratory Animal Resources (US), Committee on Care and Use of Laboratory Animals, National Institutes of Health (US), Division of Research Resources. Guide for the care and use of laboratory animals. Bethesda, MD: U.S. Department of Health and Human Services, Public Health Service, National Insititutes of Health; 1985.

17. Salehi I, Taheraslani Z, Moradkhani S. Hydro-alcoholic extract of Commiphora mukul gum resin may improve cognitive impairments in diabetic rats. Avicenna J Med Biochem 2014;2:e24906.

18. Moradkhani S, Salehi I, Abdolmaleki S, Komaki A. Effect of Calendula officinalis hydroalcoholic extract on passive avoidance learning and memory in streptozotocin-induced diabetic rats. Anc Sci Life 2015;34:156-61.

19. Omidi G, Karimi SA, Rezvani-Kamran A, Monsef A, Shahidi S, Komaki A. Effect of coenzyme Q10 supplementation on diabetes induced memory deficits in rats. Metab Brain Dis 2019;34:83340.

20. Gheibi S, Jeddi S, Carlstrom M, Gholami H, Ghasemi A. Effects of long-term nitrate supplementation on carbohydrate metabolism, lipid profiles, oxidative stress, and inflammation in male obese type 2 diabetic rats. Nitric Oxide 2018;75:27-41.

21. Ramesh B, Karuna R, Sreenivasa Reddy S, Sudhakara G, Saralakumari D. Ethanolic extract of Commiphora mukul gum resin attenuates streptozotocin-induced alterations in carbohydrate and lipid metabolism in rats. EXCLI J 2013;12:556-68,

22. Seed J, Chapin RE, Clegg ED, Dostal LA, Foote RH, Hurtt ME, et al. Methods for assessing sperm motility, morphology, and counts 
in the rat, rabbit, and dog: a consensus report. ILSI Risk Science Institute Expert Working Group on Sperm Evaluation. Reprod Toxicol 1996;10:237-44.

23. Narayana K, Prashanthi N, Nayanatara A, Kumar HH, Abhilash K, Bairy KL. Effects of methyl parathion (o,o-dimethyl o-4-nitrophenyl phosphorothioate) on rat sperm morphology and sperm count, but not fertility, are associated with decreased ascorbic acid level in the testis. Mutat Res 2005;588:28-34.

24. Bahmanzadeh M, Abolhasani F, Amidi F, Ejtemaeimehr S, Salehnia M, Abasi M. The effects of nitric oxide synthase inhibitor (L-NAME) on epididymal sperm count, motility, and morphology in varicocelized rat. DARU 2008;16:23-8.

25. La Vignera S, Vicari E, Condorelli R, D'Agata R, Calogero AE. Ultrasound characterization of the seminal vesicles in infertile patients with type 2 diabetes mellitus. Eur J Radiol 2011;80:e64-7.

26. Ding GL, Liu Y, Liu ME, Pan JX, Guo MX, Sheng JZ, et al. The effects of diabetes on male fertility and epigenetic regulation during spermatogenesis. Asian J Androl 2015;17:948-53.

27. Jangir RN, Jain GC. Diabetes mellitus induced impairment of male reproductive functions: a review. Curr Diabetes Rev 2014;10:147-57.

28. Karimi J, Goodarzi MT, Tavilani H, Khodadadi I, Amiri I. Increased receptor for advanced glycation end products in spermatozoa of diabetic men and its association with sperm nuclear DNA fragmentation. Andrologia 2012;44 Suppl 1:280-6.

29. Shrilatha B, Muralidhara. Early oxidative stress in testis and epididymal sperm in streptozotocin-induced diabetic mice: its progression and genotoxic consequences. Reprod Toxicol 2007;23: 578-87.

30. Hisatomi A, Sakuma S, Fujiwara M, Seki J. Effect of tacrolimus on the cauda epididymis in rats: analysis of epididymal biochemical markers or antioxidant defense enzymes. Toxicology 2008;243: 23-30.

31. Barve K. Herbs in the treatment of diabetes induced erectile dysfunction. J Pharm Phytother 2013;1:2-8.

32. Matsuda H, Morikawa T, Ando S, Oominami H, Murakami T, Kimura I, et al. Absolute stereostructures of polypodane-type triterpenes, myrrhanol $A$ and myrrhanone $A$, from guggul-gum resin (the resin of Balsamodendron mukul). Chem Pharm Bull (Tokyo) 2004;52:1200-3.

33. Jasuja ND, Choudhary J, Sharma P, Sharma N, Joshi SC. A review on bioactive compounds and medicinal uses of Commiphora mukul. J Plant Sci 2012;7:113-37.

34. Cornick CL, Strongitharm BH, Sassano G, Rawlins C, Mayes AE, Joseph AN, et al. Identification of a novel agonist of peroxisome proliferator-activated receptors alpha and gamma that may contribute to the anti-diabetic activity of guggulipid in Lep(ob)/ Lep(ob) mice. J Nutr Biochem 2009;20:806-15.

35. Huang TH, Teoh AW, Lin BL, Lin DS, Roufogalis B. The role of herbal PPAR modulators in the treatment of cardiometabolic syndrome. Pharmacol Res 2009;60:195-206.

36. Sharma B, Salunke R, Srivastava S, Majumder C, Roy P. Effects of guggulsterone isolated from Commiphora mukul in high fat diet induced diabetic rats. Food Chem Toxicol 2009;47:2631-9.

37. Bellamkonda R, Karuna R, Sasi Bhusana Rao B, Haritha K, Manjunatha B, Silpa S, et al. Beneficiary effect of Commiphora mukul ethanolic extract against high fructose diet induced abnormalities in carbohydrate and lipid metabolism in wistar rats. J Tradit Complement Med 2017;8:203-11.

38. Ramesh B, Karuna R, Sreenivasa RS, Haritha K, Sai MD, Sasi BR, et al. Effect of Commiphora mukul gum resin on hepatic marker enzymes, lipid peroxidation and antioxidants status in pancreas and heart of streptozotocin induced diabetic rats. Asian Pac J Trop Biomed 2012;2:895-900.

39. Mahmoud AM, Alqahtani S, Othman Sl, Germoush MO, Hussein $\mathrm{OE}$, Al-Basher G, et al. Commiphora molmol modulates glutamate-nitric oxide-cGMP and Nrf2/ARE/HO-1 pathways and attenuates oxidative stress and hematological alterations in hyperammonemic rats. Oxid Med Cell Longev 2017;2017:7369671.

40. De Lamirande E, Leclerc P, Gagnon C. Capacitation as a regulatory event that primes spermatozoa for the acrosome reaction and fertilization. Mol Hum Reprod 1997;3:175-94.

41. Sikka SC. Oxidative stress and role of antioxidants in normal and abnormal sperm function. Front Biosci 1996;1:e78-86.

42. Paick JS. Role of reactive oxygen species in male infertility. Korean J Androl 2003;21:1-11.

43. Sharma RK, Agarwal A. Role of reactive oxygen species in male infertility. Urology 1996;48:835-50.

44. Rao RM, Khan ZA, Shah AH. Toxicity studies in mice of Commiphora molmol oleo-gum-resin. J Ethnopharmacol 2001;76: 151-4.

45. Shah AH, Al-Shareef AH, Ageel AM, Qureshi S. Toxicity studies in mice of common spices, Cinnamomum zeylanicum bark and Piper longum fruits. Plant Foods Hum Nutr 1998;52:231-9. 